\title{
SOME EXAMPLES OF CRITICAL POINTS FOR THE TOTAL MEAN CURVATURE FUNCTIONAL
}

\author{
JOSU ARROYO ${ }^{1}$, MANUEL BARROS ${ }^{2}$ AND OSCAR J. GARAY ${ }^{1}$ \\ ${ }^{1}$ Departamento de Matemáticas, Universidad del País Vasco/Euskal \\ Herriko Unibertsitatea, Aptdo 644, 48080 Bilbao, Spain \\ (mtparolj@lg.ehu.es; mtpgabeo@lg.ehu.es) \\ ${ }^{2}$ Departamento de Geometría y Topología, Facultad de Ciencias, \\ Universidad de Granada, 18071 Granada, \\ Spain (mbarros@goliat.ugr.es)
}

(Received 31 March 1999)

\begin{abstract}
We study the following problem: existence and classification of closed curves which are critical points for the total curvature functional, defined on spaces of curves in a Riemannian manifold. This problem is completely solved in a real space form. Next, we give examples of critical points for this functional in a class of metrics with constant scalar curvature on the three sphere. Also, we obtain a rational one-parameter family of closed helices which are critical points for that functional in $\mathbb{C P}^{2}(4)$ when it is endowed with its usual Kaehlerian structure. Finally, we use the principle of symmetric criticality to get equivariant submanifolds, constructed on the above curves, which are critical points for the total mean curvature functional.
\end{abstract}

Keywords: total curvature; critical points; total tension

AMS 1991 Mathematics subject classification: Primary 53C40; 53A05

\section{Introduction}

The tension field of a submanifold is the Euler-Lagrange operator associated with the energy of the submanifold [9] and it is precisely the mean curvature vector field. Thus, the amount of tension that a submanifold receives at one point is measured by the mean curvature function at that point, $\alpha$. We can also measure the tension in a global manner and wonder about the minimal amount of total tension $\int \alpha$ that a submanifold receives from the surrounding space. An original argument of Pinkall [14], which involves the principle of symmetric criticality [13], has been exploited in a series of articles to obtain a sort of 'bridge' among the one-dimensional and higher-dimensional cases of several variational problems associated with functionals defined in terms of the tension. In this context, new examples of Willmore surfaces and Willmore-Chen submanifolds (for a wide class of conformal structures) starting from 'generalized' elasticae have been constructed (see, for example, $[1,2,4,6,14]$ ). Note also that the existence of closed critical points 
is of great importance in all these variational problems. Hence, we are interested in the following problem.

Problem 1.1. Existence and classification of (closed) critical points for the total curvature functional, $\mathcal{F}=\int_{\gamma} \kappa$, defined on spaces of curves in a Riemannian manifold.

In $\S 2$, the Euler-Lagrange equation for this functional is computed. Furthermore, in this section we solve the problem of existence and classification of its critical points in a real space form completely. For closed critical points the result essentially reduces to the following: the critical points for $\mathcal{F}$ in a real space form are either any closed curve in the plane or the horizontal liftings, via the usual Hopf map, in $\mathbb{S}^{3}(1)$ of certain multiple covers of closed curves in $\mathbb{S}^{2}\left(\frac{1}{2}\right)$ whose enclosed area is a rational of $\pi$.

Next, we study the existence of closed critical points for $\mathcal{F}$ in Riemannian manifolds other than real space forms. We deal with this problem in the most natural spaces after those with constant curvature. Since, in dimension three, Einstein metrics make no sense, we consider (in $\S 4$ ) a deformation of the standard metric in $\mathbb{S}^{3}(1)$ through metrics with constant scalar curvature (they constitute the so called canonical variation of $\mathbb{S}^{3}(1)$ [7]). These metrics are less rigid than the standard one; however, they still have a large group of isometries. Moreover, the holonomy of the natural connection on the Hopf fibration, is preserved. We then give a one-parameter class of metrics in $\mathbb{S}^{3}(1)$ which admit rational one-parameter families of non-geodesic closed critical points of the total curvature. These critical points are realized as closed geodesics of minimal flat tori, embedded in these spaces (see Theorem 3.3).

As we said, three is the largest dimension in which we can expect to find fully immersed critical points of the total curvature in real space forms. On the other hand, the FubiniStudy metric on $\mathbb{C P}^{2}(4)$ gives the most important four-dimensional Einstein-Riemannian space with no constant sectional curvature. In $\S 5$ we investigate the critical points of the total curvature in the two-dimensional complex projective plane endowed with the Fubini-Study metric. By means of a technique introduced in [6], consisting basically of using a new complex Frenet frame constructed via the Hopf map, we prove that every critical helix of the total curvature in the complex projective plane is the image under the natural projection of a one-parameter subgroup of $S U(3)$. This key result allows us to find a one-parameter family of critical helices to the total curvature in $\mathbb{C P}^{2}(4)$ which contains a rational indexed subfamily of closed critical points.

In the last section we apply the principle of symmetric criticality to construct equivariant submanifolds which are critical points of the total mean curvature functional, starting from closed curves which are critical points for the total curvature functional. This criterion for reduction of variables in the total mean curvature variational problem has many applications and we exhibit some of them. In particular, we obtain a rational one-parameter family of $\mathbb{S}^{1}$-invariant flat tori with non-zero constant mean curvature in the round five sphere, which are critical points for the total mean curvature functional (see Corollary 5.2).

In contrast with the above result, we obtain another family of flat tori with nonzero constant mean curvature in the round five sphere, which are critical points for this 
functional (see Proposition 5.3). To obtain this second family we use a direct method, first we compute the Euler-Lagrange equations associated with the functional and then we use some ideas from the Chen theory of finite-type submanifolds $[3,8]$.

\section{The variational problem in a real space form}

In this article we will use the notation and terminology of [12]. Let $M$ be an $n$-dimensional Riemannian manifold with metric $g=\langle\cdot, \cdot\rangle$, Levi-Civita connection $\nabla$ and curvature tensor $R$. For an immersed curve $\gamma$ in $M$, let $\kappa$ be its oriented curvature if $n=2$ and its curvature $(\kappa \geqslant 0)$ otherwise. Let $\Lambda$ be the space of closed curves immersed in $M$ if $n=2$ and the space of closed curves with a finite number of inflection points otherwise. Notice that at inflection points, $\kappa$ vanishes and so we cannot define, in general, a unit normal at those points. We consider the functional $\mathcal{F}: \Lambda \rightarrow \mathbb{R}$ defined by

$$
\mathcal{F}(\gamma)=\int_{\gamma} \kappa \mathrm{d} s .
$$

To compute the first variational formula for $\mathcal{F}$, we use a standard argument which involves some integrations by parts, the Frenet equations of $\gamma$ in $M$ which are defined up to at most a finite number of points when $n \geqslant 3$, and a technical lemma similar to Lemma 1.1 of [12]. We then obtain

$$
\begin{aligned}
\frac{\mathrm{d} \mathcal{F}}{\mathrm{d} t}(0)=\int_{\gamma}\left\langle\tau^{2} N-\tau_{s} B+\tau \eta-R(N, T) T, W\right\rangle \mathrm{d} s & \\
& \quad-\sum_{i=1}^{m}\left\langle\nabla_{T} W, N\left(s_{i}^{+}\right)-N\left(s_{i}^{-}\right)\right\rangle+\sum_{i=1}^{m}\left\langle W\left(s_{i}\right), \nabla_{T} N\left(s_{i}^{+}\right)-\nabla_{T} N\left(s_{i}^{-}\right)\right\rangle,
\end{aligned}
$$

where $T$ and $N$ stand for the unit tangent and the unit normal. The latter is well defined along $\gamma$ if $n=2$, while perhaps it is not defined in the inflection points $\gamma\left(s_{i}\right), 1 \leqslant i \leqslant m$ when $n \geqslant 3$. Also, $B$ is the unit binormal in this case, $\tau \geqslant 0$ denotes the torsion and $\tau_{s}$ its derivative with respect to the arclength parameter $s$. Finally, $\eta$ belongs to the subbundle which is normal to the one spanned by $\{T, N, B\}$, defined along $\gamma$ except at most at a finite number of points, and $W$ is a generic variational vector field along $\gamma$.

The last formula allows us to characterize the critical points for $\mathcal{F}$.

Lemma 2.1. $\gamma \in \Lambda$ is a critical point for $\mathcal{F}$ if and only if the following conditions hold:

(1) $N, B$ and $\tau$ are well defined in the inflection points of $\gamma$; and

(2) the following Euler-Lagrange equation is satisfied

$$
R(N, T) T=\tau^{2} N-\tau_{s} B+\tau \eta
$$

It should be noticed that the first condition in the statement of this lemma always holds when $n=2$. The Euler-Lagrange equation (2.2), has early but interesting consequences as follows. 
(1) If $M$ has negative sectional curvature, then it is free of non-geodesic critical points of the total curvature functional $\mathcal{F}$.

(2) If $M$ is a surface, then a curve $\gamma$ is a critical point of $\mathcal{F}$ if and only if the Gaussian curvature $G$ of $M$ vanishes identically along $\gamma$. Hence we have, for example, the following conditions.

(a) Any immersed closed curve in $\mathbb{R}^{2}$, the Euclidean plane, or in a flat torus is automatically a critical point for $\mathcal{F}$.

(b) If $M$ is a surface of revolution in $\mathbb{R}^{3}$ such that the set $\{p \in M / G(p)=0\}$ has empty interior, then the critical points for $\mathcal{F}$ are parallels made up of parabolic points. In particular, a torus of revolution in $\mathbb{R}^{3}$ has exactly two critical points for $\mathcal{F}$ corresponding to two parallels of parabolic points.

(3) Let $M^{n}$ be a real space form with sectional curvature $c$ and dimension $n \geqslant 3$. If $\gamma$ is a non-geodesic critical point for $\mathcal{F}$ which is fully immersed in $M$, then $n=3$ and $c>0$.

To complete the classification of the critical points for $\mathcal{F}$ in a real space form $M$, we only need to consider the case $M=\mathbb{S}^{3}(1)$, the round unit 3-sphere. To better understand the next theorem, we recall that the usual Hopf map $\pi: \mathbb{S}^{3}(1) \rightarrow \mathbb{S}^{2}\left(\frac{1}{2}\right)$ becomes a Riemannian submersion when the basis $\mathbb{S}^{2}\left(\frac{1}{2}\right)$ is chosen to have radius $\frac{1}{2}$.

Theorem 2.2. Let $\beta$ be an immersed closed curve in $\mathbb{S}^{3}(1)$, then $\beta$ is a critical point for $\mathcal{F}$ if and only there exists a natural number, say $m$, such that $\beta$ is a horizontal lift, via the Hopf map, of the $m$-fold cover of an immersed closed curve $\gamma$ in $\mathbb{S}^{2}\left(\frac{1}{2}\right)$, whose enclosed oriented area $A$ is a rational multiple of $\pi$ given by $A=(p / m) \pi$, where $p$ and $m$ are relative primes.

Proof. Let $\gamma$ be a closed curve in $\mathbb{S}^{2}\left(\frac{1}{2}\right)$ of length $L$ enclosing an oriented area $A \in\left[-\frac{1}{2} \pi, \frac{1}{2} \pi\right]$. If $\bar{\gamma}$ is a horizontal lift of $\gamma$, then $\bar{\gamma}(L)=\mathrm{e}^{\mathrm{i} \delta} \cdot \bar{\gamma}(0)$ for some $\delta \in[-\pi, \pi]$ (this number $\delta$ is known as the holonomy number of the canonical principal connection defined on the circle bundle $\left.\pi: \mathbb{S}^{3}(1) \rightarrow \mathbb{S}^{2}\left(\frac{1}{2}\right)\right)$. Now, $\bar{\gamma}$ closes up if and only if there exists $m \in \mathbb{N}$ such that after $m$ consecutive liftings of $\gamma$ (that means, we lift the $m$-fold cover of $\gamma$ ) we get $\bar{\gamma}(m \cdot L)=\mathrm{e}^{\mathrm{i} m \delta} \cdot \bar{\gamma}(0)=\bar{\gamma}(0)$. Then $\delta=2 \pi p / m$ for a certain integer $p$. On the other hand, a well-known argument (from [11]) can easily be adapted to give that $\delta=2 A$. Hence, $\bar{\gamma}$ closes after $m$ consecutive liftings if and only if $A=(p / m) \pi$.

Obviously a geodesic of $\mathbb{S}^{3}(1)$ is a critical point (it is a minimum) for $\mathcal{F}$ and certainly it is obtained by lifting horizontally the 2 -fold cover of a geodesic in $\mathbb{S}^{2}\left(\frac{1}{2}\right)$. Thus, in the next argument we consider non-geodesic critical points for $\mathcal{F}$. According to the EulerLagrange equation (2.2), a curve $\beta$ immersed in $\mathbb{S}^{3}(1)$ is a critical point for $\mathcal{F}$ if and only if its torsion satisfies $\tau^{2}=1$. Also, if $\bar{\gamma}$ is a horizontal lift of $\gamma$, it is not difficult to see that $\bar{\kappa}=\kappa \cdot \pi$ and $\bar{\tau}^{2}=1$, where $\bar{\kappa}, \bar{\tau}$ and $\kappa$ stand for the curvature and the torsion of $\bar{\gamma}$ and the curvature of $\gamma$, respectively. Consequently, $\beta$ and $\bar{\gamma}$ must be congruent in $\mathbb{S}^{3}(1)$ when $\gamma=\pi(\beta)$. 
To illustrate this theorem, we will exhibit some examples.

(1) A rational one-parameter family of closed helices in $\mathbb{S}^{3}(1)$ which are critical points for $\mathcal{F}$ can be obtained as follows. The enclosed area $A$ of a small circle $\gamma$ in $\mathbb{S}^{2}\left(\frac{1}{2}\right)$ of radius $r \in\left(0, \frac{1}{2}\right]$ is given by $A=\frac{1}{2} \pi\left(1-\sqrt{1-4 r^{2}}\right)$. A horizontal lift of the $m$-fold cover of $\gamma$ closes up if and only if $\frac{1}{2}\left(1-\sqrt{1-4 r^{2}}\right)=p / m$. We now solve this equation in $r$ to obtain a rational one-parameter family of radii whose circles lift to critical points for $\mathcal{F}$.

(2) A horizontal lifting in $\mathbb{S}^{3}(1)$ of the 2-fold cover of any closed free elastica in $\mathbb{S}^{2}\left(\frac{1}{2}\right)$ is a critical point for $\mathcal{F}$. In fact, closed free elasticae in $\mathbb{S}^{2}\left(\frac{1}{2}\right)$ were studied in [12]. In particular, they enclose an area $A=\frac{1}{2} \pi$, and so we only need to apply Theorem 2.2 .

(3) The spherical elliptic lemniscate. In spherical coordinates $(\phi, \theta)$ on $\mathbb{S}^{2}\left(\frac{1}{2}\right), \phi$ standing for the longitude and $\theta$ denoting the latitude, we consider the curve given by

$$
\gamma: \frac{1}{4}\left(\phi^{2}+\sin ^{2} \theta\right)^{2}=a^{2} \sin ^{2} \theta+b^{2} \phi^{2}
$$

with parameters $a$ and $b$ satisfying $b^{2} \geqslant 2 a^{2}$. This curve is the image under an appropriate Lambert projection of an elliptic lemniscate in the plane (that is the inverse curve of an ellipse of axis $2 a$ and $2 b$ with respect to its centre). Since the Lambert projection preserves the area, the area enclosed by $\gamma$ in $\mathbb{S}^{2}\left(\frac{1}{2}\right)$ is $A=\frac{1}{2}\left(a^{2}+b^{2}\right) \pi$. Now we choose $a$ and $b$ such that $a^{2}+b^{2}$ is a rational number, say $p / q$, with $a^{2}+b^{2} \leqslant 1$. Then, a horizontal lift of the $2 q$-fold cover of $\gamma$ gives a critical point for $\mathcal{F}$ in $\mathbb{S}^{3}(1)$. See figures 1 and 2 .

(4) The spherical limaçon or the spherical snail of Pascal. In the above spherical coordinates on $\mathbb{S}^{2}\left(\frac{1}{2}\right)$ we choose the curve

$$
\gamma:\left(\frac{1}{2} \phi^{2}+\frac{1}{2} \sin ^{2} \theta-2 a \phi\right)^{2}=h^{2}\left(\phi^{2}+\sin ^{2} \theta\right),
$$

for suitable parameters $a$ and $h$. This is nothing but the image under the Lambert projection of a limaçon of Pascal (the inverse curve of an ellipse with respect to a focus), see figures 3 and 4 . Therefore, $\gamma$ encloses the area $A=\left(h^{2}+\frac{1}{2} a^{2}\right) \pi$. Again, for a suitable choice of parameters $a$ and $h$, we get examples of critical points for $\mathcal{F}$ in $\mathbb{S}^{3}(1)$ by applying Theorem 2.2.

(5) The spherical folium. It can be obtained from the folium simple in the plane (see figures 5 and 6), via a Lambert projection. In spherical coordinates on $\mathbb{S}^{2}\left(\frac{1}{2}\right)$ it is defined by

$$
\gamma: \frac{1}{2}\left(\phi^{2}+\sin ^{2} \theta\right)^{2}=a \phi^{3}
$$

and it encloses an area $A=\frac{5}{32} a^{2} \pi$ on $\mathbb{S}^{2}\left(\frac{1}{2}\right)$. For example, if we choose $a=1$, then a horizontal lift of the 32 -fold cover of the corresponding spherical folium is a critical point for $\mathcal{F}$ in $\mathbb{S}^{3}(1)$.

(6) The spherical roses. Let $(\rho, \psi)$ be polar coordinates in the plane. For an integer number $m$, we consider the curve

$$
\delta: \rho=c \cdot \sin (m \psi)
$$




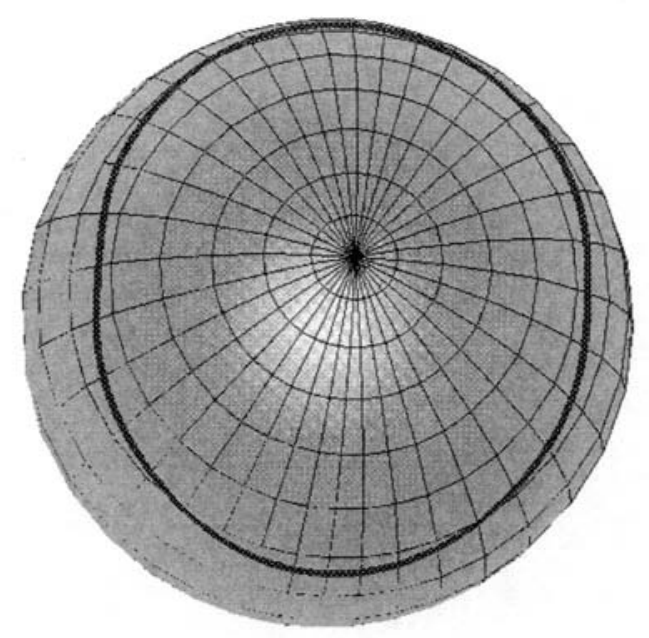

Figure 1. Spherical elliptic lemniscate for $a^{2}=\frac{1}{8} ; b^{2}=\frac{1}{4}$

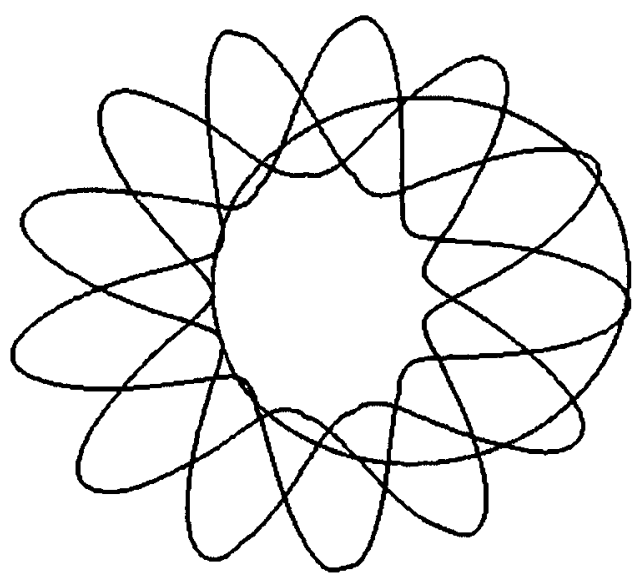

Figure 2. Stereographic projection of a closed lifting of the spherical elliptic lemniscate.

where $c$ is a real number. The curve $\delta$ defines a rose in the plane with $m$ petals if $m$ is odd and $2 m$ petals when $m$ is even. These curves are also called clover curves (for example, if $m=2$ we obtain the four-leaved clover, while if $m=3$ we get the three-leaved clover or trefoil, which are very important when plotting tensor properties of quaternary and ternary crystals). The enclosed area in the plane by a clover curve is $A=\frac{1}{4} c^{2} \pi$ if $m$ is odd and $A=\frac{1}{2} c^{2} \pi$ if $m$ is even. Now, we take the image under the Lambert projection in $\mathbb{S}^{2}\left(\frac{1}{2}\right)$ of a suitable clover curve, to obtain closed curves enclosing the same area in $\mathbb{S}^{2}\left(\frac{1}{2}\right)$. Finally, we choose $c$ such that $c^{2}$ is a rational number and apply Theorem 2.2 to have new examples of critical points for $\mathcal{F}$ in $\mathbb{S}^{3}(1)$. 


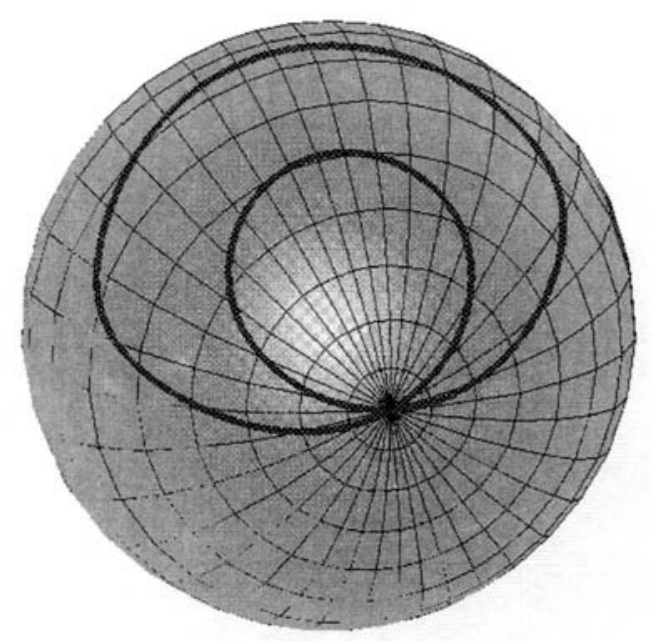

Figure 3. Spherical limaçon for $a=b=\frac{1}{4}$.

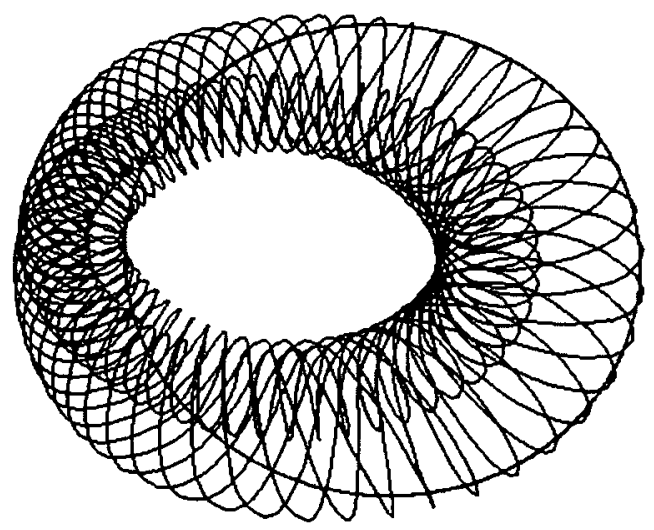

Figure 4. Stereographic projection of a closed lifting of the spherical limaçon.

\section{Critical points of the total curvature functional in non-standard 3-spheres}

The proof of Theorem 2.2 depends on two main facts. Firstly, it uses the extreme rigidity of the standard metric $\vec{g}$ on $\mathbb{S}^{3}(1)$. Secondly, it is based also on the non-trivial holonomy of the natural connection on the principal bundle $\pi: \mathbb{S}^{3}(1) \rightarrow \mathbb{S}^{2}\left(\frac{1}{2}\right)$. In this section we wish to modify this setting by studying critical points of the total curvature functional on the 3-sphere when it is endowed with metrics of lower degree of rigidity than the standard one, but still very rich in isometries. For a Riemannian submersion, one can define a deformation of the total space metric by changing the relative scales of the base and the fibres. This deformation is called the canonical variation (see [7] for details). In particular, the canonical variation of $\pi: \mathbb{S}^{3}(1) \rightarrow \mathbb{S}^{2}\left(\frac{1}{2}\right)$-or simply the canonical variation of the 


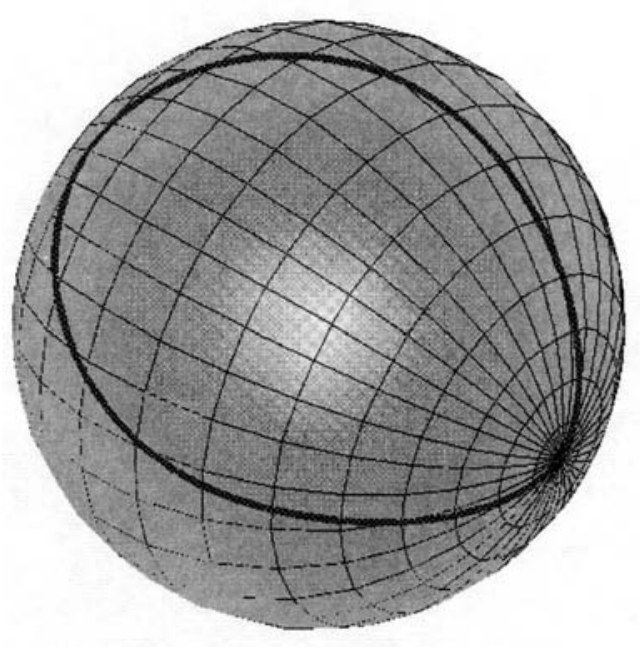

Figure 5. Spherical folium for $a=1$.

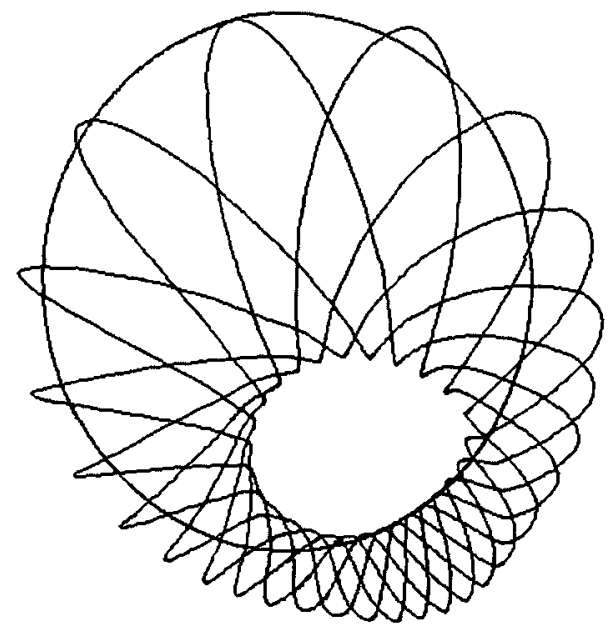

Figure 6. Stereographic projection of a closed lifting of the spherical folium.

standa:d metric, $\bar{g}$, of $\mathbb{S}^{3}(1)$--is defined to be

$$
\left\{\bar{g}_{t}=\pi^{*}(g)+t^{2} \cdot \mathrm{d} r^{2}, t>0\right\},
$$

where $\mathrm{d} r^{2}$ is the usual metric on the fibres $\mathbb{S}^{1}(1)$. Observe that $\bar{g}_{1}=\bar{g}$. Some important properties of this one-parameter family of metrics on the 3 -sphere are collected in the following lemma [7].

Lemma 3.1. We have the following. 
(1) For any $t>0, \pi:\left(\mathbb{S}^{3}, \bar{g}_{t}\right) \rightarrow\left(\mathbb{S}^{2}, g\right)$ is a Riemannian submersion with geodesic fibres. Moreover, these Riemannian submersions have the same horizontal distribution.

(2) For any $t>0$, the $\mathbb{S}^{1}$-action on $\mathbb{S}^{3}$ to obtain $\mathbb{S}^{2}$ as orbit space is performed by isometries of $\left(\mathbb{S}^{3}, \bar{g}_{t}\right)$.

(3) For any $t>0,\left(\mathbb{S}^{3}, \bar{g}_{t}\right)$ has constant scalar curvature.

Given an immersed curve $\gamma$ in $\mathbb{S}^{2}\left(\frac{1}{2}\right)$, we denote by $M_{\gamma}^{t}=\pi^{-1}(\gamma)$ its complete lift endowed with the $\bar{g}_{t}$-induced metric. These surfaces have been studied in [1] from the point of view of the Willmore conjecture and in [5] in relation to the Lawson conjecture. Next, we give some of their properties.

Lemma 3.2. The following assertions hold.

(1) Let $\gamma$ be an immersed closed curve in $\mathbb{S}^{2}\left(\frac{1}{2}\right)$ of length $L>0$ and enclosing an oriented area $A$. Then $M_{\gamma}^{t}=\pi^{-1}(\gamma)$ is a flat torus isometric to $\mathbb{R}^{2} / \Gamma_{t}$, where $\Gamma_{t}$ is the lattice spanned by $(0,2 \pi t)$ and $(L, 2 A)$. This torus is embedded if and only if $\gamma$ has no self-intersections in $\mathbb{S}^{2}\left(\frac{1}{2}\right)$.

(2) If $\bar{\gamma}(s)$ is a horizontal lift of $\gamma(s)$ (here $s$ works as the arclength parameter), then $\phi: \mathbb{R}^{2} \rightarrow M_{\gamma}^{t}=\pi^{-1}(\gamma)$ defined by $\phi(s, z)=\mathrm{e}^{\mathrm{i} z} \cdot \bar{\gamma}(s)$ is a covering map. It maps the lines parallel to the $z$-axis into the fibres (which are circles of radius $t$ ) and the $s$-lines into the horizontal lifts of $\gamma$.

(3) If $\rho$ denotes the curvature function of $\gamma$ in $\mathbb{S}^{2}\left(\frac{1}{2}\right)$, then $\xi=\phi_{s} \wedge\left((1 / t) \phi_{z}\right)=\mathrm{i} \cdot \phi_{s}$ is a unit normal vector field on $M_{\gamma}^{t}=\pi^{-1}(\gamma)$ in $\left(\mathbb{S}^{3}, \bar{g}_{t}\right)$. Moreover, the shape operator with respect to $\left\{\phi_{s}, \eta=(1 / t) \phi_{z}\right\}$ is given by

$$
\left(\begin{array}{ll}
\rho & t \\
t & 0
\end{array}\right) .
$$

(4) Let $\beta_{\theta}$ be the geodesic of $M_{\gamma}^{t}=\pi^{-1}(\gamma)$ with slope $\theta$ (slope measured with respect to the horizontal lifts of $\gamma$ ), then the Frenet apparatus $\left\{T_{\theta}, N_{\theta}, B_{\theta}, \kappa_{\theta}, \tau_{\theta}\right\}$ of $\beta_{\theta}$ in $\left(\mathbb{S}^{3}, \bar{g}_{t}\right)$ is given by

$$
\begin{aligned}
T_{\theta} & =\cos \theta \cdot \phi_{s}+\sin \theta \cdot \eta, \\
N_{\theta} & =\mathrm{i} \cdot \phi_{s}, \\
B_{\theta} & =-\sin \theta \cdot \phi_{s}+\cos \theta \cdot \eta, \\
\kappa_{\theta} & =\rho(s) \cos ^{2} \theta+t \sin 2 \theta, \\
\tau_{\theta} & =-\frac{1}{2} \rho(s) \sin 2 \theta+t \cos 2 \theta .
\end{aligned}
$$

We can use some formulae relative to Riemannian submersions (see [7, Theorems 9.28 and 9.69]) in the Euler-Lagrange equations (2.2) to see that $\beta_{\theta}$ is a critical point of the total curvature functional in $\left(\mathbb{S}^{3}, \bar{g}_{t}\right)$ if and only if its torsion and slope are related by

$$
\tau_{\theta}^{2}=\left(4-3 t^{2}\right) \cos ^{2} \theta+t^{2} \sin ^{2} \theta .
$$


Theorem 3.3. For any rational number $q$, it is possible to find a positive real number $t$, with $t^{2} \in(1,2)$, and a curve $\beta_{q}$, such that $\beta_{q}$ is a closed critical point of the total curvature functional in $\left(\mathbb{S}^{3}, \bar{g}_{t}\right)$. The curve $\beta_{q}$ appears as a closed geodesic in a minimal flat torus embedded in $\left(\mathbb{S}^{3}, \bar{g}_{t}\right)$.

Proof. First we use Lemma 3.1 to see that a geodesic of slope $\theta$ in $M_{\gamma}^{t}=\pi^{-1}(\gamma)$ is closed if and only if there exists a rational number $q$ such that

$$
\tan \theta=t \cdot\left(\frac{2 A}{L}+q \frac{2 \pi t}{L}\right)
$$

hence, choosing $\gamma$ as a geodesic of $\mathbb{S}^{2}\left(\frac{1}{2}\right)$, formula (3.8) turns into

$$
\tan \theta=t \cdot(1+2 t q)
$$

for a rational number $q$ verifying $1+2 q t \neq 0$. With this choice of $\gamma$, we combine (3.7) with (3.6) and $\rho=0$ to see that $\beta_{\theta}$ is a critical point of the total curvature functional in $\left(\mathbb{S}^{3}, \bar{g}_{t}\right)$ if and only if

$$
\cos ^{2} 2 \theta=\frac{2-t^{2}}{t^{2}}
$$

In order to obtain closed critical points $\beta_{\theta}$, we compare $\tan ^{2} \theta$ computed from (3.9) and (3.10), respectively. We have

$$
\tan ^{2} 2 \theta+\frac{4 t^{2}(1+2 q t)^{2}}{\left(1-t^{2}(1+2 q t)^{2}\right)^{2}}=\frac{2 t^{2}-2}{2-t^{2}}
$$

Consequently, we are looking for the zeros of the function

$$
F(t, q)=4 t^{2}\left(2-t^{2}\right)(1+2 t q)^{2}+\left(2-2 t^{2}\right)\left(1-t^{2}(1+2 t q)^{2}\right)^{2} .
$$

It is obvious that for any $q \in \mathbb{Q}, \lim _{t^{2} \rightarrow 1} F(t, q)>0$ and $\lim _{t^{2} \rightarrow 2} F(t, q)<0$. Hence, there exists $t>0$ with $t^{2} \in(1,2)$ such that $F(t, q)=0$. Now, for this $t$, we compute $\theta$ by using either (3.9) or (3.10), it does not matter which, and we obtain a closed critical point of the total curvature functional in $\left(S^{3}, \bar{g}_{t}\right)$.

\section{Critical points of the total curvature functional in the complex projective plane}

Let $\mathbb{C P}^{2}(4)$ be the complex projective plane endowed with its usual complex structure $J$ and the Fubini-Study metric of constant holomorphic sectional curvature 4. For an arclength immersed curve $\gamma(s)$ in $\mathbb{C P}^{2}(4)$, we choose $\left\{T, \xi_{2}, \xi_{3}, \xi_{4}\right\}$ to be a Frenet reference along $\gamma$ and denote by $\{\kappa, \tau, \delta\}$ the three curvatures of $\gamma$. It is clear that $J T=\cos \phi_{2} \xi_{2}+$ $\cos \phi_{3} \xi_{3}+\cos \phi_{4} \xi_{4}$ with $\cos ^{2} \phi_{2}+\cos ^{2} \phi_{3}+\cos ^{2} \phi_{4}=1$. In particular, $\phi_{2}$ is the angle between the complex tangent plane span $\{T, J T\}$ and the osculating plane $\operatorname{span}\left\{T, \xi_{2}\right\}$. A curve $\gamma$ is said to be of constant slant if $\phi_{2}$ is constant along $\gamma$. 
A straightforward computation involving the Euler-Lagrange equations (2.2), the Frenet equations for $\gamma$ in $\mathbb{C P}^{2}(4)$, and the expression of the curvature tensor field of $\mathbb{C P}^{2}(4)$ shows that $\gamma$ is a critical point for the total curvature functional in $\mathbb{C P}^{2}(4)$ if and only if $\{\kappa, \tau, \delta\}$ are solutions of

$$
\begin{aligned}
& 0=1-\tau^{2}+3 \cos ^{2} \phi_{2} \\
& 0=\tau^{\prime}+3 \kappa \cos \phi_{2} \cos \phi_{3} \\
& 0=\tau \delta+3 \cos \phi_{2} \cos \phi_{4}
\end{aligned}
$$

We recall that a helix in a Riemannian manifold is a curve whose Frenet curvatures are constant. The standard Frenet equations of a curve $\gamma$ into $\mathbb{C P}^{2}(4)$ are useful, for example, in defining the concept of helix. For our purposes we have need of a reference frame along the curve $\gamma$ other that the Frenet one, which involves the complex structure $J$ of $\mathbb{C P}^{2}(4)$ and the Hopf map $\pi: \mathbb{S}^{5}(1) \rightarrow \mathbb{C P}^{2}(4)$. This new frame was introduced in [6] for the study of elastic curves in $\mathbb{C P}^{2}(4)$. One way to describe this frame is to begin by lifting the curve $\gamma$ in $\mathbb{C P}^{2}(4)$ to a horizontal curve $Y(s)$ in $\mathbb{S}^{5}$. It should be noticed that this lifting is not unique, but different lifts are all of the form $\mathrm{e}^{\mathrm{i} r} \cdot Y(s), r$ being a constant. We also observe that $Y(s)$ is arclength parametrized too, because $\pi$ is a Riemannian submersion. The tangent vector $T(s)=\gamma^{\prime}(s)$ lifts to $\bar{T}(s)=Y^{\prime}(s)$. Now, we may uniquely choose a vector field $U(s)$ along $\gamma(s)$, orthogonal to $T(s)$, so that its horizontal lift $\bar{U}(s)$ gives the third component in a special unitary frame $\sigma(s)=\{Y(s), \bar{T}(s), \bar{U}(s)\}$ in $\mathbb{C}^{3}$. In other words, $\sigma(s)$ is a lifting of the curve $\gamma(s)$ to a curve in $S U(3)$. It is not difficult to see that $\sigma$ satisfies the following differential equation:

$$
\frac{\mathrm{d} \sigma(s)}{\mathrm{d} s}=\sigma(s) \cdot A(s)
$$

where $A(s)$ is a matrix in $s u(3)$. Since the curve $Y(s)$ is horizontal, then $A(s)$ must have the form:

$$
\left(\begin{array}{ccc}
0 & -1 & 0 \\
1 & \kappa_{1} \mathrm{i} & -\kappa_{2}+\kappa_{3} \mathrm{i} \\
0 & \kappa_{2}+\kappa_{3} \mathrm{i} & -\kappa_{1} \mathrm{i}
\end{array}\right)
$$

This equation can be projected down to $\mathbb{C P}^{2}(4)$. Hence, the new frame along $\gamma(s)$ is $\{T, J T, U, J U\}(s)$ and its associated equations are

$$
\begin{aligned}
\nabla_{T} T & =\kappa_{1} J T+\kappa_{2} U+\kappa_{3} J U, \\
\nabla_{T} J T & =-\kappa_{1} T-\kappa_{3} U+\kappa_{2} J U, \\
\nabla_{T} U & =-\kappa_{2} T+\kappa_{3} J T-\kappa_{1} J U \\
\nabla_{T} J U & =-\kappa_{3} T-\kappa_{2} J T+\kappa_{1} U .
\end{aligned}
$$

Notice that $\kappa_{1}^{2}+\kappa_{2}^{2}+\kappa_{3}^{2}=\kappa^{2}$. In this setting, we use once more the Euler-Lagrange equations (2.2) in $\mathbb{C P}^{2}(4)$, to see that $\gamma(s)$ is a critical point of the total curvature in 
$\mathbb{C P}^{2}(4)$ if and only if $\left\{\kappa_{1}, \kappa_{2}, \kappa_{3}\right\}(s)$ are solutions of

$$
\begin{aligned}
& 0=\left(\frac{\kappa_{1}}{\kappa}\right)^{\prime \prime}+4 \frac{\kappa_{1}}{\kappa}+\kappa_{3}\left(\frac{\kappa_{2}}{\kappa}\right)^{\prime}-\kappa_{2}\left(\frac{\kappa_{3}}{\kappa}\right)^{\prime}, \\
& 0=\left(\frac{\kappa_{2}}{\kappa}\right)^{\prime \prime}+\frac{\kappa_{2}}{\kappa}-\kappa_{3}\left(\frac{\kappa_{1}}{\kappa}\right)^{\prime}+\kappa_{1}\left(\frac{\kappa_{3}}{\kappa}\right)^{\prime}, \\
& 0=\left(\frac{\kappa_{3}}{\kappa}\right)^{\prime \prime}+\frac{\kappa_{3}}{\kappa}+\kappa_{2}\left(\frac{\kappa_{1}}{\kappa}\right)^{\prime}-\kappa_{1}\left(\frac{\kappa_{2}}{\kappa}\right)^{\prime} .
\end{aligned}
$$

Next, we will solve the following problem in the affirmative.

Problem 4.1. Do there exist closed helices in $\mathbb{C P P}^{2}(4)$ which are critical points for the total curvature functional?

The following result is the chief point in the solution.

Theorem 4.2. Every critical helix for the total curvature functional in $\mathbb{C P}^{2}(4)$ is the image, under the natural projection, of a one-parameter subgroup of $S U(3)$.

Proof. If $\gamma$ is a critical helix of $\mathcal{F}$ in $\mathbb{C P}^{2}(4)$, then it has constant slant, say $\phi_{2}=\phi$. Also, $\tau$ and $\delta$ can be expressed in terms of $\phi$ by

$$
\tau^{2}=3 \cos ^{2} \phi+1 \quad \text { and } \quad \delta=\frac{3 \cos \phi\left(1-\cos ^{2} \phi\right)^{1 / 2}}{\left(3 \cos ^{2} \phi+1\right)^{1 / 2}}
$$

We compare $\nabla_{T}^{2} T$ when it is computed using the complex and the Frenet equations, respectively, to obtain

$$
\left(\kappa_{1}^{\prime}\right)^{2}+\left(\kappa_{2}^{\prime}\right)^{2}+\left(\kappa_{3}^{\prime}\right)^{2}=\kappa^{2} \tau^{2}
$$

From $\kappa_{1}^{2}+\kappa_{2}^{2}+\kappa_{3}^{2}=\kappa^{2}$, it is clear that we can find a function $\psi(s)$, along $\gamma(s)$, such that

$$
\kappa_{1}=\kappa \cos \phi, \quad \kappa_{2}=\kappa \sin \phi \cos \psi(s), \quad \kappa_{3}=\kappa \sin \phi \sin \psi(s) .
$$

We put $\omega=\psi^{\prime}$ and then combine (4.13) with (4.14) to get

$$
\tau^{2}=\omega^{2} \sin ^{2} \phi
$$

which proves that $\omega$ is a non-zero constant.

Since $\gamma$ satisfies the Euler-Lagrange equations, we may substitute these into equations (4.9)-(4.11). The resulting equations are dependent and can be simplified to

$$
\begin{aligned}
C & =\frac{\omega^{2}-1}{\omega}, \\
S^{2} & =\frac{4\left(\omega^{2}-1\right)}{\omega^{2}},
\end{aligned}
$$

where $C$ and $S$ are constants given by

$$
\kappa_{1}=C, \quad \kappa_{2}=S \cos \omega s, \quad \kappa_{3}=S \sin \omega s .
$$


Thus, by using (4.14), (4.15), (4.18) and (4.12), we have that the other constants can also be expressed in terms of $\omega$ :

$$
\begin{aligned}
\kappa^{2} & =2+\omega^{2}-\left(3 / \omega^{2}\right), \\
\tau^{2} & =\frac{4 \omega^{2}}{\left(\omega^{2}+3\right)} \\
\delta^{2} & =\frac{9 \omega^{4}-2 \omega^{2}+1}{\left(\omega^{2}-1\right)\left(\omega^{2}+3\right)}, \\
S^{2} & =\kappa^{2}-C .
\end{aligned}
$$

Note from (4.19) that $\omega^{2} \in(1, \infty)$.

The differential equation (4.4) giving the lift $\sigma(s)$ of $\gamma$ to $S U(3)$ may be now written as

$$
\sigma^{\prime}(s)=\sigma(s) \cdot\left(\begin{array}{ccc}
0 & -1 & 0 \\
1 & C \mathrm{i} & -S \mathrm{e}^{-\mathrm{i} \omega s} \\
0 & S \mathrm{e}^{\mathrm{i} \omega s} & -C \mathrm{i}
\end{array}\right) .
$$

We define a new curve in $S U(3)$, say $\tilde{\sigma}(s)=(\tilde{Y}, \tilde{Z}, \tilde{U})(s)$ by

$$
\tilde{\sigma}(s)=\sigma(s) \cdot\left(\begin{array}{ccc}
\mathrm{e}^{-\mathrm{i} \omega s / 3} & 0 & 0 \\
0 & \mathrm{e}^{-\mathrm{i} \omega s / 3} & 0 \\
0 & 0 & \mathrm{e}^{2 \mathrm{i} \omega s / 3}
\end{array}\right) .
$$

We observe that $\tilde{Y}(s)=\mathrm{e}^{-\mathrm{i} \omega s / 3} \cdot Y(s)$ and so $\Pi(\tilde{Y}(s))=\Pi(Y(s))=\gamma(s)$. That means that $\tilde{Y}(s)$ is a lift of $\gamma(s)$, although it is not a horizontal curve. The advantage of using $\tilde{\sigma}(s)$ is that it satisfies the following system of differential equations with constant coefficients:

$$
\tilde{\sigma}^{\prime}(s)=\tilde{\sigma}(s) \cdot M,
$$

where $M \in s u(3)$ is given by

$$
M=\left(\begin{array}{ccc}
-\frac{1}{3} \mathrm{i} \omega & -1 & 0 \\
1 & \left(C-\frac{1}{3} \omega\right) \mathrm{i} & -S \\
0 & S & \left(\frac{2}{3} \omega-C\right) \mathrm{i}
\end{array}\right) .
$$

Corollary 4.3. There exists a one-parameter family of critical helices of the total curvature living fully into $\mathbb{C P}^{2}(4)$. For any choice of $\omega^{2} \in(0, \infty)$, we have a critical helix of the total curvature in $\mathbb{C P}^{2}(4)$ with curvatures given by formulae (4.19)-(4.22), and slant given by

$$
\cos ^{2} \phi=\frac{\left(\omega^{2}-1\right)}{\left(\omega^{2}+3\right)} \in(0,1) \text {. }
$$


It remains to examine which among the above critical helices are closed. It is enough, of course, for the lifted curve $\tilde{\sigma}(s)$ to be a closed curve in $S U(3)$. Therefore, we must find a positive number, say $L$, so that $\tilde{\sigma}(s+L)=\tilde{\sigma}(s)$. Since $\tilde{\sigma}(s)=\mathrm{e}^{s M}$ is a one-parameter subgroup of $S U(3)$, this reduces to the problem of finding $L>0$ such that the eigenvalues of $L \cdot M$ are all integer multiples of $2 \pi \mathrm{i}$. Let $t_{1}, t_{2}, t_{3}$ be the eigenvalues of $M$. Since $M$ is in $s u(3)$, we have $t_{1}+t_{2}+t_{3}=0$. It follows that the required condition for the roots is that $t_{2} / t_{1}$ be rational.

The characteristic equation of $M$ can be written as

$$
r^{3}-\frac{1}{3}\left(9+\omega^{2}\right) r-\frac{2}{27}\left(9+\omega^{2}\right) \omega=0 .
$$

The roots of this equation turn out to be

$$
\begin{aligned}
& r_{1}=\frac{1}{3}\left(\sqrt{\omega^{2}+9}\right) \cos \left(\frac{1}{3} \theta\right), \\
& r_{2}=\frac{1}{3}\left(\sqrt{\omega^{2}+9}\right) \cos \left(\frac{1}{3}(\theta+2 \pi)\right), \\
& r_{3}=\frac{1}{3}\left(\sqrt{\omega^{2}+9}\right) \cos \left(\frac{1}{3}(\theta+4 \pi)\right),
\end{aligned}
$$

where

$$
\cos \theta=\frac{-\omega}{\sqrt{\omega^{2}+9}}, \quad \sin \theta=\frac{3}{\sqrt{\omega^{2}+9}} .
$$

As a consequence, we have the following theorem.

Theorem 4.4. Let $q$ be a rational number with $\sqrt{3}<q<3$. Define an angle $\theta$, $\frac{3}{4} \pi<\theta<\pi$, by $q=\sqrt{3} \tan \frac{1}{3} \theta$. Then for $\omega=-3 \cot \theta$, the corresponding critical helix of the total curvature in $\mathbb{C P}^{2}(4)$ is closed.

\section{Equivariant critical points of the total mean curvature functional}

Let $G$ be a $q$-dimensional, compact Lie group endowed with a bi-invariant metric $\mathrm{d} \sigma^{2}$ and let $p: P \rightarrow M$ be a principal fibre $G$-bundle with a principal connection 1 -form $\omega$. For any Riemannian metric $g$ on $M$, we can find one and only one Riemannian metric $\bar{g}$ on $P$ such that $p:(P, \bar{g}) \rightarrow(M, g)$ is a Riemannian submersion with totally geodesic fibres isometric to $\left(G, \mathrm{~d} \sigma^{2}\right)$ and horizontal distribution associated with $\omega$. This metric can be defined as

$$
\bar{g}=p^{*}(g)+\omega^{*}\left(\mathrm{~d} \sigma^{2}\right)
$$

The natural action of $G$ on $P$ is carried out by isometries of $(P, \bar{g})$. Let $\gamma$ be a curve immersed in $M$, then its complete lift $N_{\gamma}=p^{-1}(\gamma)$ is a $(q+1)$-dimensional submanifold in $P$ which is $G$-invariant. Furthermore, any $(q+1)$-dimensional $G$-invariant submanifold in $P$ is obtained in this way. This is the chief point that allows us to apply the principle of symmetric criticality (see [13]) to obtain the following criterion for reduction of variables.

Proposition 5.1. $N_{\gamma}=p^{-1}(\gamma)$ is a critical point of the total mean curvature functional in $(P, \bar{g})$ if and only if $\gamma$ is a critical point of the total curvature functional in $(M, g)$. 
Proof. According to the principle of symmetric criticality, $N_{\gamma}=p^{-1}(\gamma)$ is a critical point of the total mean curvature functional in $(P, \bar{g})$ if and only if it is a critical point of that functional but restricted to the submanifold of $G$-invariant immersions. To compute this restriction, we only need to consider the following relationship between the mean curvature $\alpha$ of $N_{\gamma}=p^{-1}(\gamma)$ in $(P, \bar{g})$ and the curvature $\kappa$ of $\gamma$ in $(M, g)$, which was given in [1]:

$$
\alpha=(1 / q+1)(\kappa \circ p)
$$

We can combine the last proposition with the results explained in $\S 2$ to obtain interesting consequences. Next we display some of them.

(1) If $(M, g)$ is a real space form of dimension $n$, then $N_{\gamma}=p^{-1}(\gamma)$ is a critical point of the total mean curvature functional in $(P, \bar{g})$ if and only if either

(a) $(M, g)$ is flat and $\gamma$ is any closed curve immersed in a totally geodesic surface of $(M, g)$, or

(b) the Riemannian universal covering of $(M, g)$ is a round $n$-sphere, say of radius one, $\mathbb{S}^{n}(1)$. Moreover, up to covering maps, $\gamma$ must be contained in some $\mathbb{S}^{3}(1)$ and it must be obtained according to Theorem 2.2.

(2) Let $M$ be a genus-one compact surface and denote by $\tilde{M}$ a regular covering of $M$. Let $H$ be the deck transformation group. Obviously, $\tilde{M}$ can be regarded as a principal fibre $H$-bundle which admits a trivial flat connection $\omega$. Let $h$ be a monomorphism from $H$ into $G$. The transition functions of the above $H$-bundle can be extended, via $h$, to $G$-functions, which can be chosen as transition functions of a principal fibre $G$-bundle, say $p: P \rightarrow M$, on $M$. Also, the connection $\omega$ can be used to define a flat connection $\omega$ on this. Then we have the following.

(a) If $(M, g)$ is a flat torus, then any $N_{\gamma}=p^{-1}(\gamma)$ is a critical point for the total mean curvature functional in $(P, \bar{g})$.

(b) If $g$ is chosen such that $(M, g)$ is an anchor ring, then $(P, \bar{g})$ has exactly two $G$ invariant submanifolds which are critical points for the total mean curvature functional.

We can also combine Theorem 4.4 with Proposition 5.1, to obtain the following corollary.

Corollary 5.2. There exists a rational one-parameter family of $\mathbb{S}^{1}$-invariant tori, which are critical points for the total mean curvature functional in the round five sphere.

Now, we use a direct method to obtain critical points for the total mean curvature functional in the round five sphere (compare with [3]). In contrast with Corollary 5.2, the following examples cannot be obtained as liftings, via the Hopf map, of curves in $\mathbb{C P}^{2}(4)$. In order to do this, first we compute the first variation of the total mean curvature defined 
on the space of immersions of a compact surface in $\mathbb{S}^{5}(1)$. We use a standard argument which essentially involves some integrations by parts (see [16] for a similar computation) to obtain the following Euler-Lagrange equations,

$$
\Delta H-\tilde{A}(H)+4 \alpha^{2} H=0,
$$

where $H$ is the mean curvature vector field, $\Delta$ denotes the Laplacian associated with the normal connection, and $\tilde{A}$ is the Simons operator [15].

Since minimal surfaces are trivial examples of critical points for the total mean curvature functional, we will construct examples of non-minimal flat tori which are critical points for this functional in $\mathbb{S}^{5}(1)$. Given three real numbers $a, b$ and $c$ with $a, b>0$, we consider the lattice $\Gamma$ in $\mathbb{R}^{2}$ spanned by $(2 \pi a, 2 \pi c)$ and $(0,2 \pi b)$. We choose $n, m, \bar{n} \in$ $\mathbb{Z}-\{0\}$ such that $\omega=(n / a)-(m c / b a)$ and $\bar{\omega}=\bar{n} a$ satisfy $\omega \neq \bar{\omega}$ and $\omega \neq 0$, we also consider that $m / b>1$. Then we define $y: \mathbb{R}^{2} \rightarrow \mathbb{C}^{3}$ by

$$
y(s, t)=\left(r \cos \frac{t}{r} \mathrm{e}^{\mathrm{i} \omega s}, r \sin \frac{t}{r} \mathrm{e}^{\mathrm{i} \omega s}, \sqrt{1-r^{2}} \mathrm{e}^{\mathrm{i} \bar{\omega} s}\right),
$$

where $r=b / m$. Now, this is an isometric immersion if and only if $r^{2} \omega^{2}+\left(1-r^{2}\right) \bar{\omega}^{2}=1$. Furthermore, in this case, it induces an isometric immersion $x$ from the flat torus $T=$ $\mathbb{R}^{2} / \Gamma$ in $\mathbb{S}^{5}(1)$. Some interesting properties of these immersions are collected below.

(1) The centre of mass of $x$ in $\mathbb{C}^{3}$ coincides with the centre of $\mathbb{S}^{5}(1)[8]$.

(2) The immersion $x$ is not minimal in $\mathbb{S}^{5}(1)$. In fact, it is constructed in $\mathbb{C}^{3}$ by using eigenfunctions for the Laplacian of $T$ associated with two different eigenvalues, namely $\omega^{2}+\left(1 / r^{2}\right)$ and $\bar{\omega}^{2}$. Therefore, we say that it is of 2-type in the sense of Chen [8].

(3) These immersions have non-zero constant mean curvature.

(4) They are not invariant under the natural $\mathbb{S}^{1}$-action on $\mathbb{S}^{5}(1)$ to obtain $\mathbb{C P}^{2}(4)$ as space of orbits.

Finally, a straightforward long computation gives the following proposition.

Proposition 5.3. The immersion $x: T \rightarrow \mathbb{S}^{5}(1)$ is a critical point for the total mean curvature functional, that is it is a solution of (5.1) if and only if the following equation holds

$$
r^{2}\left(1-2 r^{2}\right) \omega^{4}+\left(4 r^{2}-3\right) \omega^{2}+\left(2-r^{2}\right)=0 .
$$

Acknowledgements. We express our hearty thanks to David Singer for his useful comments during the elaboration of this work. This research was partly supported by a DGICYT grant PB97-0784. O.J.G. was also supported by MEC grant PR97 Programa Sectorial. 


\section{References}

1. M. Barros, Willmore tori in non-standard 3-spheres, Math. Proc. Camb. Phil. Soc. 121 (1997), 321-324.

2. M. Barros, Free elasticae and Willmore tori in warped product spaces, Glasgow Math J. 40 (1988), 263-270.

3. M. Barros AND B. Y. Chen, Stationary 2-type surfaces in a hypersphere, J. Math. Soc. Japan 39 (1987), 627-648.

4. M. BARRos and O. J. GARAY, Hopf submanifolds in $S^{7}$ which are Willmore-Chen submanifolds, Math. Z. 228 (1998), 121-129.

5. M. BARRos, A. FERrÁndez AND P. LUCAS, Lawson's type problems in non-standard 3-spheres, Q. J. Math. Oxford 50 (1999), 385-388.

6. M. Barros, O. J. Garay and D. Singer, Elasticae with constant slant in $\mathbb{C P}^{2}(4)$ and new examples of Willmore tori in $S^{5}$, Tôhoku Math. J. 51 (1999), 177-192.

7. A. L. BESSE, Einstein manifolds (Springer, 1987).

8. B.-Y. CHEN, Total mean curvature and submanifolds of finite type (World Scientific, Singapore, 1984).

9. J. Eells AND L. Lemaire, A report on harmonic maps, Bull. Lond. Math. Soc. 10 (1978), $1-68$.

10. J. ERbaChER, Reduction of codimension of an isometric immersion, J. Diff. Geom. 5 (1971), 333-340.

11. W. Greub, S. Halperin and R. Vanstone, Connections, curvature and cohomology (3 vols) (Academic Press, 1972, 1973, 1976).

12. J. Langer and D. A. Singer, The total squared curvature of closed curves, J. Diff. Geom. 20 (1984), 1-22.

13. R. S. Palais, Critical point theory and the minimax principle, Global Analysis, Proc. Symp. Pure Math. 15 (1970), 185-212.

14. U. PINKalL, Hopf tori in $S^{3}$, Invent. Math. 81 (1985), 379-386.

15. J. Simons, Minimal varieties in Riemannian manifolds, Ann. Math. 88 (1968), 62-105.

16. J. L. WEINER, On a problem of Chen, Willmore, et al., Indiana Univ. Math. J. 27 (1978), 19-35. 